\title{
An archaeal histone-like protein regulates gene expression in response to salt stress
}

\author{
Saaz Sakrikar ${ }^{1,2}$, Amy Schmid ${ }^{1,2,3^{*}}$ \\ ${ }^{1}$ Biology Department, Duke University, Durham, NC, 27708 USA \\ ${ }^{2}$ University Program in Genetics and Genomics, Duke University, Durham, NC, 27708 USA \\ ${ }^{3}$ Center for Genomics and Computational Biology, Duke University, Durham, NC, 27708 USA
}

*To whom correspondence should be addressed:

Amy Schmid

amy.schmid@duke.edu 


\begin{abstract}
Histones, ubiquitous in eukaryotes as DNA-packing proteins, find their evolutionary origins in archaea. Unlike the characterized histone proteins of a number of methanogenic and themophilic archaea, previous research indicated that HpyA, the sole histone encoded in the model halophile Halobacterium salinarum, is not involved in DNA packaging. Instead, it was found to have widespread but subtle effects on gene expression and to maintain wild type cell morphology; however, its precise function remains unclear. Here we use quantitative phenotyping, genetics, and functional genomic to investigate HpyA function. These experiments revealed that HpyA is important for growth and rod-shaped morphology in reduced salinity. HpyA preferentially binds DNA at discrete genomic sites under low salt to regulate expression of ion uptake, particularly iron. HpyA also globally but indirectly activates other ion uptake and nucleotide biosynthesis pathways in a salt-dependent manner. Taken together, these results demonstrate an alternative function for an archaeal histone-like protein as a transcriptional regulator, with its function tuned to the physiological stressors of the hypersaline environment.
\end{abstract}




\section{INTRODUCTION}

Phylogenetic analysis has shown that the histone fold domain originated in the Archaea (1-3). Histone proteins play a vital role in genome compaction and regulation of gene expression in eukaryotes (4). The four core eukaryotic histones (H3, H4, H2A, H2B) share a histone fold domain, which is involved in histone dimerization and DNA-binding (5-7). Proteins containing the histone fold are present in all known major archaeal lineages (7). Archaeal histone-like proteins have been most extensively characterized in species representing the euryarchaeal superphylum, with most work so far focusing on Methanothermus fervidus (8) and Thermococcus kodakarensis (9-11). From in vitro and in vivo studies, including structural analyses $(12,13)$ and nuclease digestion (14), it was interpreted that archaeal histones function similarly to those of eukaryotes. These histones appeared to act as the major chromatin protein by forming extended polymeric structures that wrap DNA in multiples of 30-60 bp. Like eukaryotic histones, in some species archaeal histones have been shown to influence global transcription levels by hindering initiation (15) or elongation (16). Archaeal histones can also inhibit the binding of site-specific transcription factors (TFs) through competition (17). These studies led to the current prevailing hypothesis that archaeal histone function largely resembles that of eukaryotes in terms of genome compaction and gene expression, although some have noted key differences $(7,18)$.

Recent evidence in other model systems challenge this hypothesis. For example, a deletion mutant of the sole histone of Methanosarcina mazei was viable, but exhibited reduced growth when exposed to radiation (19). Phylogenetics and molecular dynamics simulations in other model methanogens that encode multiple histone variants suggest various functions in the chromatin environment (20). Previous work from our group demonstrated an alternative regulatory function for HpyA, the sole histone of the hypersaline-adapted species Halobacterium salinarum (21). Like in M. mazei, HpyA is dispensable for cell viability. Unlike in M. mazei histone, HpyA is important for maintaining wild type gene expression and cell shape under optimum growth conditions. HpyA protein levels were too low to facilitate genome-wide DNA compaction (21). Phylogenetic and proteomics evidence in hyperthermophiles suggests that chromatin compaction allows DNA stability to prevent unwanted transcription by promoter melting at high temperature (22). Histone point mutants that cannot compact DNA exhibit differential expression of specific genomic regions (11). Together these findings challenge a predominant hypothesis, instead suggesting diverse histone functions across archaeal lineages selected for by the diverse and sometimes extreme environments of archaea. However, the function of histone-like proteins in hypersaline-adapted archaea remains understudied relative to other archaeal lineages.

Halophilic archaea have adapted to survive extreme osmotic pressure (up to $5 \mathrm{M} \mathrm{NaCl}$ ) in their natural salt lake environments by counterbalancing with up to $4 \mathrm{M}$ potassium ions in the cytoplasm (23). Due to the resultant highly ionic cytoplasm, haloarchaeal proteins have evolved a negatively charged surface (24), including the histone protein. This is in contrast to all other known species, where the positively charged surface of histones facilitates DNA-histone interactions $(3,21)$. It has previously been observed in vitro that naked DNA under high salinity tends to spontaneously form structures similar to the beads-on-a-string observed with histonebound DNA (25), calling into question the need for protein-based genome compaction. Based on these results and given the unusual chemistry of the haloarchaeal saturated salt cytoplasm, here we hypothesize that the non-canonical function of HpyA in gene regulation is linked to the unique hypersaline cytoplasmic environment of $\mathrm{Hbt}$. salinarum. 
We tested this hypothesis using a battery of in vivo quantitative phenotyping and functional genomics assays. Growth rate and cell morphology in low sodium was impaired in the $\Delta h p y A$ deletion strain, confirming a link between the gene and salt concentration. Protein-DNA binding assays (ChIP-seq) revealed reproducible, salt-dependent, genome-wide binding of HpyA at nearly 60 discrete sites -- a binding pattern too infrequent to coat or compact the genome. However, the high prevalence of binding within gene bodies suggests that the mechanism of regulation differs substantially from that of canonical TFs. Integration of DNA binding with transcriptomics data revealed direct regulation of iron uptake by HpyA. Global, indirect regulation of transport of other ions, biosynthesis of purines, and DNA replication and repair was also observed. Together, these results suggest that HpyA functions as a specific, direct transcriptional regulator of metal ion balance. HpyA thereby maintains growth rate and rodshaped cell morphology during hypo-osmotic stress.

\section{MATERIALS AND METHODS}

\section{Strains, media and general culturing:}

Strains used in this study have been described in Dulmage et al 2015 (21), summarized in Table S1. All strains were constructed from a Halobacterium salinarum NRC-1 background with ura3 (encodes uracil biosynthesis functions) gene deleted to enable uracil counterselection (26). Growth assays were carried out using strain MDK407 ( $\Delta u r a 3)$ as the parent strain (control, referred to here as wild type, or WT) and KAD100 ( $\Delta$ ura3 $\Delta h p y A)$ as the $\Delta h p y A$ deletion strain.

For ChIP-seq, strains carrying the hpyA gene tagged at its C-terminus with the HA epitope were used (21). The control strain was AKS134 ( $\triangle$ hpyA deletion carrying the empty vector pMTFCHA). The experimental strain was KAD128, which contained the pKAD17 plasmid expressing HpyA-HA driven by its native promoter (primers and plasmids given in Table S1). pKAD17 was generated by: (a) insertion of $h p y A$ into the pMTF-cHA plasmid upstream of the $\mathrm{HA}$ tag sequence (between the Ndel and Hindlll restriction sites); and (b) replacement by isothermal ligation of the $P_{\mathrm{fdx}}$ promoter of the plasmid with the $P_{\text {rpa200 }}$ native promoter sequence of HpyA at the Kpnl site.

The media used for all experiments was $\mathrm{Hbt}$. salinarum complete media (CM) containing $250 \mathrm{~g} / \mathrm{L}$ $\mathrm{NaCl}, 20 \mathrm{~g} / \mathrm{L} \mathrm{MgSO} 4 \bullet 7 \mathrm{H}_{2} \mathrm{O}, 3 \mathrm{~g} / \mathrm{L}$ trisodium citrate dihydrate, $2 \mathrm{~g} / \mathrm{L} \mathrm{KCl}, 10 \mathrm{~g} / \mathrm{L}$ Bacteriological peptone (Oxoid). $\mathrm{pH}$ was adjusted to 6.8 . Media were supplemented with $50 \mathrm{\mu g} / \mathrm{mL}$ uracil to compensate for the uracil auxotrophy of $\Delta u r a 3$ parent and derivative strains. Reduced salt media was made identically except for $\mathrm{NaCl}$, which was reduced to $199 \mathrm{~g} / \mathrm{L}$ (3.4 M). For plasmid strains, $1 \mu \mathrm{g} / \mathrm{mL}$ mevinolin (AG Scientific) was added to liquid medium and $2.5 \mu \mathrm{g} / \mathrm{mL}$ to solid media to maintain selective pressure on the plasmid.

Cells were routinely streaked fresh from frozen stock onto solid medium. Individual colonies were picked from plates and inoculated into $5 \mathrm{~mL} \mathrm{CM} \mathrm{(with} \mathrm{additives} \mathrm{when} \mathrm{necessary)} \mathrm{and}$ allowed to grow for approximately 4 days at $42^{\circ} \mathrm{C}$ in a shaking incubator until stationary phase was reached. These starter cultures were diluted by sub-culturing to OD600 0.02 into $50 \mathrm{~mL}$ of media indicated in the figures and grown until harvesting as described below.

\section{Growth and microscopy:}

For growth curve phenotyping, 9 biological replicates of $\Delta u r a 3$ (MDK407) and $\Delta h p y A$ (KAD100) strains were cultured in $125 \mathrm{~mL}$ flasks at $42^{\circ} \mathrm{C}$ in a shaking incubator. Optical density (OD) 
measurements were taken at time zero, then at 3-4 hour intervals following the initial lag phase of $\sim 12$ hours. Raw growth data are provided in Table S2. Resultant growth curves were fit by logistic regression to calculate instantaneous growth rate $\left(\mu_{\max }\right)$ using the $\mathrm{R}$ package grofit $(27)$. The code for analysis and visualization of these growth data are contained in https://github.com/amyschmid/HpyA_codes.

For microscopy, cultures of $\Delta u r a 3, \Delta h p y A$, and $\triangle h p y A$ / pKAD17 (strain KAD128) were each grown to mid-exponential phase. $8 \mu \mathrm{l}$ aliquots were placed on a thin, flat, agarose pad impregnated with 4.3M NaCl as described (28). Cells were imaged at $100 \mathrm{X}$ using a Zeiss Axio Scope A1 microscope with a Pixelink PL-E421M camera. Images were analyzed for circularity using the MicrobeJ package within the ImageJ software (29). Given that circularity distributions were skewed, adjusted bootstrap percentile corrected $95 \%$ confidence intervals were calculated by 1,000 -fold ordinary non-parametric bootstrap resampling of the median with replacement. The boot() package in the $\mathrm{R}$ coding environment was used for these calculations.

\section{ChIP-seq experiments:}

One biological replicate colony of AKS134 (Empty vector control) and four replicates of KAD128 (expressing HpyA-HA) were cultured as described above. The $50 \mathrm{~mL}$ cultures were grown in $125 \mathrm{~mL}$ flasks and their growth was monitored by OD600 until the time for harvesting (exponential phase: $36-50$ hours, OD 0.2-0.35, growth rate $\sim 0.032 \mathrm{hr}^{-1}$; stationary phase: $\sim 70$ 140 hours, OD 1.4-1.7, growth rate $\sim 0.017 \mathrm{hr}^{-1}$ ). Strains were PCR-checked for the presence of the plasmid expressing hpyA-HA prior to each experiment (see Table $\mathbf{S 1}$ for primers).

Harvested cells $(45 \mathrm{~mL})$ were immediately cross-linked using $1.4 \mathrm{~mL} 37 \%$ formaldehyde (final concentration $=1 \% \mathrm{v} / \mathrm{v}$ ) and immuoprecipitated as described in Wilbanks et al, $2012(30)$, with certain modifications to the protocol: the cross-linking reaction was allowed to proceed for 20 minutes, and cell pellets were resupended in $800 \mu \mathrm{L}$ lysis buffer. Resulting DNA was extracted with Phenol:Chloroform:Isoamyl alcohol (25:24:1) and then ethanol precipitation. Library preparation and single-end sequencing was carried out by the Duke Center for Genomic and Computational Biology Sequencing and Genomic Technologies core facility using the Illumina HiSeq4000 instrument.

\section{Analysis of ChIP-seq data:}

Gzipped FastQ files (Accession: PRJNA703048, GEO: GSE182514) were analyzed using FastQC software. Information provided as input included read sequence quality, length distribution, and presence of adapters. Adapters were trimmed from the reads using Trim Galore!, and these trimmed sequences were aligned to the Hbt. salinarum NRC-1 genome (RefSeq ID GCF_000006805.1, assembly ID ASM680v1) to generate a SAM file using Bowtie2 with default parameters. End-to-end alignment was suitable for trimmed reads (31). FastQC and Trim Galore! are available online at http://www.bioinformatics.babraham.ac.uk/projects/ (2015 version). The SAM files were converted to binary (BAM), sorted and indexed using SAMtools (32). Sorted BAM files were used for peak calling. WIG files for easy visualization were also generated using SAMtools, with coverage recorded every $10 \mathrm{bp}$. All code used to analyze ChIPseq data are available in File S1 at https://github.com/amyschmid/HpyA_codes.

The sorted BAM files were used for peak-calling with MACS2 (33) version 2.1.1 callpeak function. Parameters were: nomodel, qval $=0.05$ cutoff. Called peaks were combined across replicates using the multiBedIntersect function of the bedtools package (34). Only peaks 
detected in at least two biological replicate experiments were kept in downstream analyses. Genes within 500 bp of these reproducible peaks were annotated using the IRanges package in $R$ (35). Resultant peaks were then manually curated to remove the following: (a) false positives caused by local variability in input control sequencing read depth; (b) local duplications and deletions associated with transposases and integrases; (c) one peak that was also detected in the HA tag-alone input control; (d) peaks located nearby redundant genes. Details of the code and dependencies for the entire workflow for peak calling and visualization are noted in the github repository https://github.com/amyschmid/HpyA_codes.

Resultant peaks were then classified based on their genomic locations and context to tabulate the results given in Figure 4 and the corresponding text (details in Table S3). Promoters were defined as the region from $500 \mathrm{bp}$ upstream of the translation start site [many halophile transcripts are leaderless (36)]. To classify binding locations "genic" or "promoter", the number of bp in the overlap between the ChIP-seq peak chromosomal coordinates and the genomic feature was calculated. If the peak overlapped both a genic and a promoter feature, the peak was classified as located within the feature with the largest overalp. Both features were counted in the case of ties. The code used to make this classification is in

https://github.com/amyschmid/HpyA codes. Operons were computationally predicted using the Operon-Mapper tool (37) and integrated with empirical predictions from Koide et al (36). Classification of TrmB binding locations are given directly in reference (38) and significance of enrichment was computed using the hypergeometric test in R. Classification and computation of enrichment $p$-values for RosR binding locations [from reference (39)] were computed using BEDtools "fisher" function (40).

\section{RNA-seq experiments:}

Six biological replicate cultures of strains MDK407 (parent) and KAD100 ( $h p p y)$ were cultivated as described above in either optimal salt $(4.2 \mathrm{M} \mathrm{NaCl})$ or low salt $(3.4 \mathrm{M} \mathrm{NaCl})$ media. Growth was monitored using OD600 until harvesting (exponential phase was defined as: 31-34 hours of growth, OD 0.1-0.4 depending on the strain and medium).

A 4.2mL aliquot of each culture was removed and centrifuged for $30 \mathrm{~s}$ at $21,000 \mathrm{xg}$ in an Eppendorf tabletop centrifuge. The supernatant was discarded and the cell pellet was immediately plunged into liquid nitrogen and stored $1-7$ days at $-80^{\circ} \mathrm{C}$. Extraction of RNA from these pellets was carried out using the Agilent Absolutely RNA Miniprep kit following the manufacturer's protocol, with an extended on-column DNase incubation of 45-60 min. Resultant RNA samples were checked for: (a) genomic DNA contamination using PCR with 200ng input RNA and 35 amplification cycles using primers listed in Table S1; (b) concentration using 260/280 nm ratio in a Nanodrop spectrophotometer; (c) quality using the Agilent Bioanalyzer RNA Nano 6000 chip (RNA Integrity Number (RIN) > 9.0). For each strain and condition, rRNA was removed from 3 replicates with NEBNext Bacteria rRNA Depletion Kit (New England Biolabs), while the other 3 were treated with NEBNext Depletion Core Reagent Set using custom probes targeted to Haloferax volcanii rRNA (Martinez-Pastor and Sakrikar, unpublished). These custom probes were designed using the NEBNext Custom RNA Depletion Design Tool (https://depletion-design.neb.com/). rRNA depletion was verified using the Bioanalyzer RNA chip. The NEBNext Ultra II Directional RNA Library Prep Kit for Illumina was used for preparing sequencing libraries, and cDNA libraries were quality-checked using the High-Sensitivity DNA Bioanalyzer chip. Paired-end sequencing was carried out at the Duke 
Center for Genomic and Computational Biology Sequencing core facility using the Novaseq6000 instrument (Illumina).

\section{RNA-seq data analysis:}

For analysis of sequencing data, paired FastQ files were trimmed and checked for quality using Trim Galore! (http://www.bioinformatics.babraham.ac.uk/projects/) and aligned to the genome using Bowtie2 (31). SAMtools was used to generate, sort, and index BAM files (32). The count function of HTSeq (41) was used to create a file assigning the number of reads to each gene (see File S1 within the Github repository for details). Outlier samples were removed from further analysis using Strong PCA (42) (https://github.com/amyschmid/HpyA codes). The R package DESeq2 (43) was used to normalize counts and batch correct across replicates for each strain and genotype (using DEseq2 default parameters). Significant differential gene expression analysis using DESeq2 applied three pairwise contrasts: $\triangle h p y A$ vs WT in optimal salt, $\Delta h p y A$ vs WT in reduced salt, and reduced vs optimal salt in a WT background. For each contrast, reproducibility and quality was checked across replicates using dispersion, MA, and volcano plots. For each contrast, Benjamini-Hochberg (BH) adjusted (44) Wald test $p<0.05$ (default within DESeq2) was used as the criterion for significant differential expression (results in Table S4).

Averaged normalized counts across biological replicates for each strain and stress treatment were then mean and variance standardized and subjected to Kmeans clustering using the factoextra package in $\mathrm{R}$, which also determines the best value for $\mathrm{K}$ (45). Resultant gene clusters were then subclustered using Kmeans and visualized using ggplot2 (46) and pheatmap (47) functions in R (https://github.com/amyschmid/HpyA codes). This clustering procedure was carried out twice, once with genes differentially expressed in both reduced and optimal salt, and then excluding genes differentially expressed in optimal salt. Results of the clustering are given in Table S5. For analysis of gene functional enrichments, the hypergeometric test $p$-value of enrichment for differentially expressed genes was calculated. Resultant $p$-values were $\mathrm{BH}$ corrected for multiple hypothesis testing. The archaeal Clusters of Orthologous Genes (arCOG) functional ontology was used for functional assignments (48), results are listed in Table S6.

\section{RESULTS}

\section{HpyA is important for wild type growth and morphology in low salinity stress conditions.}

To test the hypothesis that HpyA plays a role in salt stress, we compared the growth rate of $\mathrm{Hbt}$. salinarum $\Delta$ ura3 (parent strain, hereafter referred to as wild type or "WT") to $\triangle h p y A$ cells in rich complete medium with salt concentrations supporting optimal growth $(\mathrm{CM}, 4.2 \mathrm{M} \mathrm{NaCl})$ and $\mathrm{CM}$ with reduced salt $(3.4 \mathrm{M} \mathrm{NaCl})$. As expected from previous observations (21), instantaneous growth rate $\left(\mu_{\max }\right)$ under optimal salt of the WT strain was statistically indistinguishable from that of $\Delta h p y A$ (Fig. 1A, Table S2, Fig. S1). Reduced salt slows the instantaneous growth rate $\left(\mu_{\max }\right)$ of WT cultures to $89 \%$ of that in standard conditions. In contrast, $\Delta h p y A$ cultures show significant growth impairment in reduced salt relative to WT, growing at $67 \%$ of their standard rate. (Fig. 1B; unpaired two-sample $t$-test $p<0.008$ ).

Cell morphology of $\mathrm{Hbt}$. salinarum changes from rod-shaped to circular in the presence of low salt $(49,50)$. Our previous work demonstrated that the $\triangle h p y A$ strain exhibits similar circularity in 
standard conditions (21). To further test the hypothesis that HpyA plays a role in the salt stress response, we used phase contrast microscopy to visualize the combined effects of reduced salt and $h p y A$ deletion on cell shape. From the images, we quantified circularity of individual cells (where 1 indicates a perfectly circular cell). In media containing optimal salt concentrations, WT cells are primarily rod-shaped, whereas the $\Delta h p y A$ cells are significantly rounder (Fig. 2, nonparameteric bootstrapped $95 \%$ confidence intervals of the medians of these distributions do not overlap, see Methods). In reduced salt, WT cell morphology was more circular: the distribution was indistinguishable from that of $\triangle h p y A$ in optimal salt. $\triangle h p y A$ morphology in reduced salt was the most circular of all strain-by-genotype combinations, indicating that this strain's morphology is strongly impacted by reduced salt.

Growth and morphology defects are significantly complemented by expression of $h p y A$ from its native promoter in trans on a plasmid $(\triangle h p y A+h p y A-H A$, Fig. 2). Whole genome resequencing of the $\triangle h p y A$ strain also demonstrated that: (a) second site suppressor mutations were absent; and (b) deletion of $h p y A$ was complete through all chromosomal copies (Table S1). Hbt. salinarum is highly polyploid (51), which necessitates validation that all gene copies have been deleted. These results indicate that $\triangle h p y A$ phenotypes are solely attributable to the deletion of hpyA.

Because cell shape differences can lead to alterations in light scattering in a spectrophotometer (52), as a control, we calculated CFU/mL by dilution plate counting. We found that OD600 measurements were well correlated with CFU counts for both strains and media preparations. In optimal salt, WT cultures CFU to OD Spearman correlation was $\rho=0.81(p=0.00081), \Delta h p y A \rho$ $=0.64(p=0.015)$. In reduced salt, WT correlation was $\rho=0.93\left(p<2.2 \times 10^{-16}\right), \Delta h p y A \rho=$ $0.78(p=0.001$; Fig. S2). This indicates that the $\triangle$ hpyA growth defect observed in reduced salt (as measured by optical density) is due to differences in growth and not an artefact of the shape change. Taken together, these batch culture (Fig. 1) and single cell microscopy (Fig. 2) quantitative phenotype data suggest that HpyA is important for maintaining wild type morphology and growth in response to hypo-osmotic salt stress.

\section{HpyA binds genome-wide in a salt-specific manner}

To determine which genes are potential targets of transcriptional regulation by HpyA, we performed genome-wide DNA binding location analysis using chromatin immunoprecipitation coupled to sequencing (ChIP-seq). For this purpose, we generated an $\Delta h p y A$ strain expressing in trans HpyA translationally fused at its $\mathrm{C}$-terminus to the hemagglutinin (HA) epitope tag. This fusion construct was driven by its native promoter (see Methods and Table S1 for strain details). As described above, expression of HpyA-HA in trans complemented the circularity defect of $\triangle h p y A$, demonstrating that $\mathrm{HA}$ tag and plasmid-based expression does not interfere with wild type function of HpyA (Fig. 2). Based on the $\triangle$ hpyA phenotypes observed (Figs. $1 \&$ 2), the ChIP-seq experiments were performed at both physiological and reduced salt concentrations in both mid-exponential and stationary phase. HpyA binding was enriched relative to the background input control at a total of 59 discrete genomic locations (ChIP-seq peaks) across all conditions tested (Table S3). These 59 peaks were consistently detected in reduced salt across growth phases and biological replicate experiments (Figure 3A and B), but only 5 of these peaks remained bound in optimal salt conditions. Of the low salt peaks, 35 were detected exclusively during exponential growth phase, 14 exclusively during stationary phase, and 9 across both growth phases (Figure 3C). Because HpyA binds DNA primarily under low salt 
conditions, these results corroborate the growth and morphological impairments of $\Delta h p y A$ cells observed in early log phase under reduced salt conditions (Figure 2).

HpyA binding peaks were located nearby 86 genes (within the gene coding region or $500 \mathrm{bp}$ upstream of the gene start in the promoter / non-coding region). Few of the HpyA binding sites are located within non-coding or promoter regions of the genome $(12.9 \%, p=0.593)$. In contrast, other previously characterized $\mathrm{Hbt}$. salinarum TFs bind in a sequence-specific manner with significant preference non-coding regions [Fig. 4, (38,39)]. Binding of HpyA is also not statistically enriched for binding withing gene coding regions $(p=0.152)$. This high number was as expected because, like many archaeal genomes, the Hbt. salinarum genome is dense with coding sequences $(86 \%)$. Taken together these DNA binding results suggest that, unlike canonical histone proteins of eukaryotes and other archaeal species, HpyA binds in a saltspecific manner to a restricted set of sites genome-wide. However, unlike canonical TFs, HpyA binds apparently without preference for coding vs non-coding regions.

\section{HpyA functions primarily as an activator of genes encoding ion transport and metabolic proteins.}

Based on the quantitative phenotyping and ChIP-seq data, we reasoned that HpyA may regulate gene expression in response to salt stress. To test this hypothesis, we performed transcriptome profiling experiments in WT vs $\Delta h p y A$ strains in both optimal and reduced salt using RNA-seq (see Methods). In the WT strain, over one-third of the genes in the transcriptome were significantly differentially expressed during exponential growth phase in reduced salt compared to optimal salt conditions ( $p<0.05$; 882 genes; $37 \%$ of genome; Table S4). Of the 37 genes previously identified by microarray analysis (53), 22 genes were also identified as significantly differentially expressed in the current dataset. For these 22 genes, the fold-change in expression was strongly and significantly correlated across the two datasets $\left(\rho=0.86, p<2.2 \times 10^{-16}\right)$. Our results therefore recapitulate but also extend previous observations that $\mathrm{Hbt}$. salinarum mounts a strong, reproducible, and global regulatory response to hypo-osmotic stress.

To determine the extent of HpyA's regulatory reach, gene expression ratios ( $\triangle h p y A: W T)$ were calculated during mid-exponential growth in optimal salt and reduced salt conditions (in two separate DEseq2 analyses, see Methods). A total of 168 differentially expressed genes (DEG) were detected, 143 of which were significantly altered in reduced salt and 46 in optimal salt in $\Delta h p y A$ vs WT (Figure 5A, Table S4). Of these, 121 genes were uniquely differentially expressed in response to low salt. These genes are significantly enriched for a wide variety of functions critical to maintaining cell growth and physiology in adverse conditions, especially ion transport and nucleotide metabolism (hypergeometric test $p<0.05$ enrichment in arCOG categories(48), Figure 5B, Table S6). Across both optimum and reduced salt conditions, the expression of 21 genes was significantly affected by hpyA deletion. These genes encode predicted functions in DNA recombination, replication, and repair pathways including RadA, DNA topoisomerase VI, and RPA family proteins (Table S4, Table S6).

To determine the role of HpyA in the activation or repression of these genes, we performed Kmeans clustering analysis of normalized read count data for gene expression across the four conditions tested ( $\triangle h p y A$ in low salt, $\triangle h p y A$ in optimal salt, WT in low salt, WT in optimal salt, details in Materials and Methods). We first analyzed the expression of the 21 genes that are differentially regulated the $\triangle h p y A$ strain in both optimal and reduced salt conditions. These 21 
genes fall into 2 clear categories - 10 genes downregulated in the $\Delta h p y A$ strain and 11 genes upregulated (Fig 6A, Table S5). As noted above, genes across these two clusters are significantly enriched for DNA recombination, replication, and repair functions (8 genes). HpyA binding was detected in ChIP-seq by only one of these genes (ssb, encoding single-stranded DNA binding protein, Table S7). HpyA binding was not detected for the 20 other genes in this cluster, indicating indirect regulation by HpyA.

A separate clustering analysis of the 122 genes differentially expressed only in reduced salt in $\triangle h p y A$ yielded two main patterns (Figure 6B, Table S5). In cluster 1, genes are elevated in expression in the $\triangle h p y A$ background under reduced salt relative to WT, whereas cluster 2 genes are downregulated. Cluster 2 includes $64 \%$ of genes differentially expressed in low salt, suggesting HpyA functions as an activator in the majority of cases in reduced-salt conditions.

To more clearly observe the gene expression patterns and the function of differentially expressed genes, we further divided these two main clusters, resulting in a total of 4 subclusters (Figure 6B, Table S5). Subcluster 1.1 contains 11 genes whose expression pattern is downregulated in WT in reduced salt but upregulated in $\triangle h p y A$. This cluster includes 3 genes predicted to encode ion transport proteins (chloride, iron, and other metals). Subcluster 1.2 contains 28 genes that are upregulated in reduced salt in WT but more heavily upregulated in reduced salt in the knockout strain. The function of genes in subcluster 1.2 are varied and not statistically enriched for a particular function. However, notable among genes in cluster 1.2 include transcription factor $B$ (TFB), four amino acid biosynthesis genes, and HelA ATPdependent DNA helicase (Figure 6A, Table S5). ChIP-seq enrichment for HpyA binding was not detected nearby any of the genes in subclusters 1.1 and 1.2, suggesting indirect regulation (Figure 6B, Table S7). HpyA is therefore necessary but not sufficient for repression of cluster 1 in low salt conditions.

Cluster 2 contains many genes encoding transporters ( 21 genes across both subclusters 2.1 and 2.2). Notably, genes encoding known metal cation transporters exhibit tight clustering with their cognate transcriptional regulators SirR and VNG0147C $(54,55)$ (Table S5). Subcluster 2.1 consists of 37 genes modestly upregulated in reduced salt in the WT but strongly downregulated in reduced salt in the $\triangle h p y A$ mutant. Interestingly, ChIP-seq enrichment for HpyA binding was detected at 4 sites nearby genes in this subcluster (Table S7). Three of these 4 sites are nearby genes involved in maintenance of iron levels (Fig. 6C). These encode the siderophore (iron chelator) biosynthesis and transport operon, the Suf iron-sulfur cluster biosynthesis and transport system, and a putative oxidoreductase (VNG0405C). Surprisingly, the siderophore biosynthesis operon is bound at both the 5' and 3' ends by HpyA, which is associated with significant activation of this operon in low salt conditions (Fig. 6C, top panel). These results indicate that HpyA is required for direct activation of iron uptake under low sodium.

In subcluster 2.2, 46 genes are downregulated or constitutive across optimal and reduced salt in the WT, but more heavily downregulated in $\Delta h p y A$ in reduced salt. All 8 differentially expressed nucleotide metabolism genes are found within this subcluster, and all encode de novo purine biosynthesis enzymes. However, only one of the 46 genes of subcluster 2.2 is a direct target of HpyA (VNG0161G, encoding glutamate dehydrogenase). This suggests that HpyA regulates purine biosynthesis and other functions in this subcluster in an indirect manner. 
Together, these transcriptome profiling data integrated with ChIP-seq binding locations suggest an important role for HpyA as specific, direct activator of iron uptake, and an indirect global regulator of ion transport and nucleotide biosynthesis during hypo-osmotic stress.

\section{DISCUSSION}

Here we integrate quantitative phenotyping and functional genomics data to demonstrate that the sole histone-like protein encoded in the hypersaline adapted archaeal species $\mathrm{Hbt}$. salinarum directly activates ion uptake transporters under hypo-osmotic stress. HpyA also functions as an indirect, global activator of genes encoding functions central to cellular physiology in low ionic strength medium. These transcriptional effects enable cells to maintain rod-shaped cellular morphology and growth in hypo-osmotic conditions.

Two of the five operons under the direct transcriptional control of HpyA encode transmembrane ABC transporters that are predicted to import iron. One operon (VNG0524G-VNG0527C) encodes a putative iron-sulfur (Fe-S) cluster assembly system of the Suf family. The predicted encoded proteins exhibit moderate identity to the well-characterized $E$. coli Fe-S assembly proteins SufC, SufB, and SufD $(45 \%, 56 \%$, and $28 \%$, respectively (56)). The other operon (gabT/bdb/iucABC, VNG6210-VNG6216) encodes siderophore biosynthesis and uptake. Siderophores are high-affinity iron binding chelators that are secreted from the cell and then imported via a dedicated ABC transporter (57). In Hbt. salinarum and many bacteria, in addition to the $A B C$ transporter, this operon includes a novel L-2,4-diaminobutyrate decarboxylase (DABA DC; encoded by gabT) and a DABA aminotransferase (encoded by $b d b$ ) for siderophore biosynthesis in lieu of synthesis via polyamines (57). Because amino acids are precursors for DABA biosynthesis, down-regulation of iucABC in the $\triangle h p y A$ mutant strain may also explain the indirect differential expression of amino acid biosynthesis genes during hypo-osmotic stress.

$H b t$. salinarum is a facultative anaerobe capable of aerobic and anaerobic respiratory metabolism (58). Across the tree of life, including $\mathrm{Hbt}$. salinarum, iron is an essential cofactor for the function of respiratory complexes in the oxygen-accepting electron transport chain (59). Because reduced salinity increases oxygen saturation in the medium, these conditions would favor aerobic respiratory metabolism over anaerobic metabolism, increasing the cellular demand for iron (60). Indeed, we observe that these iron transport systems are induced in an HpyA-dependent manner under low salt conditions (Figure 6B). Low levels of iron transport expression in the $\triangle h p y A$ strain would therefore be expected to lead to low intracellular iron levels. Low intracellular iron has also been observed previously for strains deleted for idr2, which encodes a DtxR family iron-dependent TF in Hbt. salinarum. This TF also functions as a direct activator of the iucABC siderophore biosynthesis and transport operon $(54,61)$. Idr2 is a member of a complex network of TFs that regulate the response to iron imbalance $(54,61)$ and the current study suggests that HpyA is also involved in regulation of iron uptake. This mechanism of transcriptional regulation by HpyA explains the $\triangle h p y A$ growth impairment observed in low sodium conditions tested here (Fig 1).

The remaining three operons under direct HpyA regulation encode central metabolic functions (Table S7). HpyA activates glutamate dehydrogenase and acyl-coA ligase enzymes, encoded by the $g d h B$ / alkK operon. These enzymes control the entry of glutamate into the TCA cycle via the conversion of glutamate to 2-oxoglutarate. Glutamate is also a key precursor for 
biosynthesis of many metabolites, including purines and other amino acids (62,63). Direct control of this operon may explain the indirect transcriptional dysregulation of these pathways in the $\triangle h p y A$ mutant strain. HpyA activates an oxidoreductase gene (NAD-dependent epimerase predicted to act on nucleotide-sugar substrates; VNG0405C) and glycerol dehydrogenase gene and its associated operon (VNG0161G / VNG0162G), also encoding key components of core metabolism. The gene encoding a single-stranded DNA binding protein ( $s s b)$ is the only direct target predicted to be regulated by HpyA under both optimal and low salt conditions, and repressed rather than activated. Although the precise relationship between these HpyA regulatory targets and the $\triangle h p y A$ growth defect remains unclear, current knowledge of metabolism in $\mathrm{Hbt}$. salinarum suggests that, in the $\triangle h p y A$ mutant, disruption in the levels of key metabolic intermediates (glycerol, glutamate) may contribute to the growth impairment of this strain under low salt conditions.

Dysregulation of import and/or efflux of other ions (divalent metal cations, chloride, and other transporters) in the $\triangle h p y A$ mutant may also explain the cell shape change in this strain (Fig 2). The proteinaceous surface layer (S-layer) is a key cell shape determinant of $\mathrm{Hbt}$. salinarum $(64,65)$. The S-layer is pliable and allows for changes in cell shape under physical pressure and low salinity $(21,49)$. This shape change is exacerbated in $\Delta h p y A$ (Fig 2), which we hypothesize is due to dysregulation of ion transport expression. Iron has also been shown to impact cell morphology in the related haloarchaeal species Haloferax volcanii, although the underlying mechanism remains unknown (66). Expression of other pathways, for example, the S-layer (encoded by csg) and glycosylation enzymes (VNG0140G; Fig 6 and Table S4) is reduced in the $\triangle h p y A$ mutant under low salt conditions. However, these appear to play a more minor role in the $\triangle h p y A$ morphology defect given that: (a) these genes are indirect targets of HpyA regulation; and (b) overall S-layer glycosylation is unaffected in strains deleted of $h p y A$ (21). Taken together, these data suggest that HpyA salt-dependent regulation of ionic balance is a major contributor to maintainance of wild type cell morphology and growth in reduced sodium environments.

Apart from these cases of direct regulation by HpyA, the majority of differentially expressed genes are located $>500$ bp away from HpyA binding sites. This can be explained in a number of ways. Several TFs are differentially expressed in the $\Delta h p y A$ strain relative to WT in low salt (Table S4, Figure 6). Therefore, the proximate cause of indirect differential gene expression can be inferred based on prior knowledge of the global gene regulatory network (GRN) in this organism $(39,67,68)$. For example, the general TF, TfbB, is differentially expressed in $\Delta h p y A$ in low salt (cluster 1.1, Fig 6), and most of the genes in this cluster are indirectly regulated. TfbB is a direct regulator of several of the genes in this cluster, including cysK (68). Hbt. salinarum encodes 7 paralogs of transcription factor B (TFB) (69). Together, TFB and TATA binding protein recruit RNA polymerase to core promoters to initiate transcription (70). The TFB network in $\mathrm{Hbt}$. salinarum is highly interconnected: for example, TfbB directly activates TfbG, which in turn regulates other genes indirectly regulated by HpyA (e.g. metal transporter VNG1744H, Table S4). Other indirect regulation by HpyA can be attributed to metal-responsive TFs. For example, SirR and VNG0147C, members of cluster 2.1, have previously been experimentally characterized as regulators of operons encoding metal transporters, specifically manganese uptake (ZurA) and the heavy metal efflux $(Z n t A)$, respectively $(54,55)$. Aside from indirect regulation as part of a transcriptional network, we note that our data do not exclude the possibility that HpyA may function as a co-regulator, perhaps by binding DNA through interaction with another TF. Hence, we propose that HpyA may, in part, achieve its global, 
indirect regulatory effect via its regulation of genes encoding other TFs and/or through proteinprotein interaction with other sequence-specific TFs.

In addition to transcriptional regulation of ion balance, HpyA may play other functional roles during hypo-osmotic stress. More than $40 \mathrm{HpyA}$ binding sites were detected with no corresponding significant change in gene expression in the $\triangle h p y A$ knockout (Table S3, S7). HpyA prefers to bind neither coding nor non-coding genomic regions, setting it apart from characterized haloarchaeal TFs that function by canonical, sequence-specific DNA binding to promoter regions [TrmB (38) or RosR (39), Fig. 4]. We provide evidence of direct regulation both among targets bound in promoter and genic regions (Table S7). Direct regulation of expression via binding in gene bodies has also been reported for $E$. coli regulator RutR (71). Our data do not rule out that non-canonical binding modes of HpyA could also influence other aspects of the transcription cycle, including elongation or termination. Bacterial nucleoid associated proteins (NAPs) bind DNA to regulate gene expression, remodel chromatin by bending or wrapping, and/or protect the nucleoid during stress $(22,72)$. For example, the E. coli transcription regulator CRP can function both as a canonical TF (site-specific gene regulation) for some genes, and as a DNA-bending chromatin remodeler at other genomic sites $(22,72)$. These newly-discovered and expanding roles for DNA binding proteins calls for a broader perspective on the function of transcriptional regulators $(22,72)$. Likewise, further research is needed to explore such functional roles for HpyA.

Taken together, the results presented here strongly suggest that HpyA functions as a direct activator of iron regulatory genes and a global indirect regulator of diverse pathways. This function is markedly different than other characterized histones in archaea and eukaryotes.

\section{DATA AVAILABILITY}

Raw sequencing data (ChIP-seq, RNA-seq, and $\triangle h p y A$ whole genome resequencing) are freely available through the National Center for Biotechnology Information (NCBI) accession PRJNA 703048. ChIP-seq and RNA-seq metadata and additional information can be found on the Gene Expression Omnibus (GEO) through accession GSE182514. All code and input data are available on GitHub at https://github.com/amyschmid/HpyA_codes. 


\section{SUPPLEMENTARY DATA}

Figure S1: No significant difference in growth rate was observed for WT (blue) and KO (red) strains grown in optimal salt. Y-axis indicates maximum instantaneous growth rate $\left(\mu_{\max }\right)$. Crossbars indicate the median of 9 biological replicate trials.

Figure S2: OD600 is correlated with $\mathrm{CFU} / \mathrm{mL}$ across strains and conditions. To calculate colony forming units (CFU), six biological replicate cultures in selected growth phases (exponential and late exponential) were diluted $1 \times 10^{-4}$ to $1 \times 10^{-6}$ depending on the OD600. $100 \mu \mathrm{L}$ aliquots of each dilution were spread on CM plates. The number of colonies was counted after 7-10 days of growth in a $42^{\circ} \mathrm{C}$ incubator, and was related to the measured OD600 at the time of plating. In each subpanel, log2 OD600 (x-axis) is plotted against the log10 colony forming units (CFU) / $\mathrm{mL}$ (y-axis) for each of the $\triangle h p y A$ knockout $(\mathrm{KO})$ and $\Delta u r a 3$ parent strain (a.k.a. wild type, WT) in optimal salt complete medium (CM) or reduced salt medium (M) as indicated in each plot title. Spearman's correlation coefficients for OD vs CFU/mL and $p$-values of significance of each correlation are indicated at the top of each plot. Significant correlation between OD and CFU/mL is observed for each strain and condition, indicating strong correspondence between these two measures of growth, and therefore validating spectrophotometric measurements of growth.

Table S1: Strains and primers used.

Table S2: Raw growth data for parental and $\triangle h p y A$ cells in optimal and reduced salt (9 biological replicates), measured as optical density (OD600).

Table S3: List of peaks obtained by HpyA ChIP-seq; arranged by peak (S3_simplified) and by overlap of peak and genomic feature (S3_full).

Table S4: List of genes differentially expressed in $\triangle h p y A$ in reduced and optimal salt conditions.

Table S5: List of genes in each subcluster obtained by clustering of expression patterns of differentially expressed genes.

Table S6: arCOG enrichments for genes nearest the HpyA ChIP-seq peaks, and for genes differentially expressed in $\triangle h p y A$

Table S7: List of ChIP-seq peaks within 500 bp of differentially expressed genes.

\section{ACKNOWLEDGEMENTS}

We thank current and former Schmid lab members: Cynthia Darnell, Rylee Hackley, Mar Martinez-Pastor, Angie Vreugdenhill, Peter Tonner, Sungmin Hwang, and Preeti Bhanap for technical assistance with experimental methods and analysis, and for comments on the manuscript. Saaz Sakrikar is grateful to his graduate thesis committee members (David Macalpine, Amy Grunden, Richard Brennan, and Raluca Gordan) for mentorship and comments on the manuscript. We thank Deyra Rodriguez and New England Biolabs for providing reagents and advice for rRNA depletion and RNA library preparation. We thank Antoine Hocher and Tobias Warnecke for scientific discussions during the preparation of the manuscript. We thank the Duke Sequencing and Genomic Technologies Core Facility for their technical expertise in generating the sequencing data reported here. 


\section{FUNDING}

Funding for this study was provided by grants from NSF MCB 1651117, 1936024, and 1615685 to A.K.S.; and NIH T32 training grant 5T32GM007754 to the Duke University Program in Genetics and Genomics.

\section{CONFLICTS OF INTEREST}

None declared.

\section{REFERENCES}

1. Malik, H.S. and Henikoff, S. (2003) Phylogenomics of the nucleosome. Nat Struct Mol Biol, 10, 882-891.

2. Brunk, C.F. and Martin, W.F. (2019) Archaeal Histone Contributions to the Origin of Eukaryotes. Trends in Microbiology, 27, 703-714.

3. Sandman, K. and Reeve, J.N. (2006) Archaeal histones and the origin of the histone fold. Current Opinion in Microbiology, 9, 520-525.

4. Talbert, P.B. and Henikoff, S. (2010) Histone variants ancient wrap artists of the epigenome. Nat Rev Mol Cell Biol, 11, 264-275.

5. Mariño-Ramírez, L., Kann, M.G., Shoemaker, B.A. and Landsman, D. (2005) Histone structure and nucleosome stability. Expert Review of Proteomics, 2, 719-729.

6. Arents, G. and Moudrianakis, E.N. (1993) Topography of the histone octamer surface: repeating structural motifs utilized in the docking of nucleosomal DNA. Proc Natl Acad Sci U S A, 90, 10489-10493.

7. Laursen, S.P., Bowerman, S. and Luger, K. (2021) Archaea: The Final Frontier of Chromatin. J Mol Biol, 433, 166791.

8. Sandman, K., Krzycki, J.A., Dobrinski, B., Lurz, R. and Reeve, J.N. (1990) HMf, a DNAbinding protein isolated from the hyperthermophilic archaeon Methanothermus fervidus, is most closely related to histones. Proc Natl Acad Sci U S A, 87, 5788-5791.

9. Cubonovaa, L., Katano, M., Kanai, T., Atomi, H., Reeve, J.N. and Santangelo, T.J. (2012) An archaeal histone is required for transformation of Thermococcus kodakarensis. J Bacteriol, 194, 6864-6874.

10. Mattiroli, F., Bhattacharyya, S., Dyer, P.N., White, A.E., Sandman, K., Burkhart, B.W., Byrne, K.R., Lee, T., Ahn, N.G., Santangelo, T.J. et al. (2017) Structure of histone-based chromatin in Archaea. Science, 357, 609-612.

11. Sanders, T.J., Ullah, F., Gehring, A.M., Burkhart, B.W., Vickerman, R.L., Fernando, S., Gardner, A.F., Ben-Hur, A. and Santangelo, T.J. (2021) Extended Archaeal HistoneBased Chromatin Structure Regulates Global Gene Expression in Thermococcus kodakarensis. Front Microbiol, 12, 681150.

12. Decanniere, K., Babu, A.M., Sandman, K., Reeve, J.N. and Heinemann, U. (2000) Crystal structures of recombinant histones HMfA and HMfB from the hyperthermophilic archaeon Methanothermus fervidus. Journal of Molecular Biology, 303, 35-47.

13. Mattiroli, F., Bhattacharyya, S., Dyer, P.N., White, A.E., Sandman, K., Burkhart, B.W., Byrne, K.R., Lee, T., Ahn, N.G., Santangelo, T.J. et al. (2017) Structure of histone-based chromatin in Archaea. Science, 357, 609.

14. Tomschik, M., Karymov, M.A., Zlatanova, J. and Leuba, S.H. (2001) The Archaeal Histone-Fold Protein HMf Organizes DNA into Bona Fide Chromatin Fibers. Structure, 9 , 1201-1211.

15. Sun, F. and Huang, L. A euryarchaeal histone modulates strand displacement synthesis by replicative DNA polymerases. Science China Life Sciences, 59, 709-716. 
16. Xie, Y. and Reeve, J.N. (2004) Transcription by an Archaeal RNA Polymerase Is Slowed but Not Blocked by an Archaeal Nucleosome. Journal of Bacteriology, 186, 3492.

17. Wilkinson, S.P., Ouhammouch, M. and Geiduschek, E.P. (2010) Transcriptional activation in the context of repression mediated by archaeal histones. Proceedings of the National Academy of Sciences, 107, 6777.

18. Maruyama, H., Harwood, J.C., Moore, K.M., Paszkiewicz, K., Durley, S.C., Fukushima, H., Atomi, H., Takeyasu, K. and Kent, N.A. (2013) An alternative beads-on-a-string chromatin architecture in Thermococcus kodakarensis. EMBO Rep, 14, 711-717.

19. Weidenbach, K., Glöer, J., Ehlers, C., Sandman, K., Reeve, J.N. and Schmitz, R.A. (2008) Deletion of the archaeal histone in Methanosarcina mazei Gö1 results in reduced growth and genomic transcription. Molecular Microbiology, 67, 662-671.

20. Stevens, K.M., Swadling, J.B., Hocher, A., Bang, C., Gribaldo, S., Schmitz, R.A. and Warnecke, T. (2020) Histone variants in archaea and the evolution of combinatorial chromatin complexity. Proceedings of the National Academy of Sciences, 117, 33384.

21. Dulmage, K.A., Todor, H. and Schmid, A.K. (2015) Growth-Phase-Specific Modulation of Cell Morphology and Gene Expression by an Archaeal Histone Protein. mBio, 6.

22. Holowka, J. and Zakrzewska-Czerwinska, J. (2020) Nucleoid Associated Proteins: The Small Organizers That Help to Cope With Stress. Front Microbiol, 11, 590.

23. Wagner, G., Hartmann, R. and Oesterhelt, D. (1978) Potassium uniport and ATP synthesis in Halobacterium halobium. Eur J Biochem, 89, 169-179.

24. Ng, W.V., Kennedy, S.P., Mahairas, G.G., Berquist, B., Pan, M., Shukla, H.D., Lasky, S.R., Baliga, N.S., Thorsson, V., Sbrogna, J. et al. (2000) Genome sequence of Halobacterium species NRC-1. Proc Natl Acad Sci U S A, 97, 12176-12181.

25. Eickbush, T.H. and Moudrianakis, E.N. (1978) The compaction of DNA helices into either continuous supercoils or folded-fiber rods and toroids. Cell, 13, 295-306.

26. Peck, R.F., DasSarma, S. and Krebs, M.P. (2000) Homologous gene knockout in the archaeon Halobacterium salinarum with ura3 as a counterselectable marker. Mol Microbiol, 35, 667-676.

27. Kahm, M., Hasenbrink, G., Lichtenberg-Fraté, H., Ludwig, J. and Kschischo, M. (2010) grofit: Fitting Biological Growth Curves with R. 2010, 33, 21.

28. Darnell, C.L., Zheng, J., Wilson, S., Bertoli, R.M., Bisson-Filho, A.W., Garner, E.C. and Schmid, A.K. (2020) The Ribbon-Helix-Helix Domain Protein CdrS Regulates the Tubulin Homolog ftsZ2 To Control Cell Division in Archaea. mBio, 11, e01007-01020.

29. Ducret, A., Quardokus, E.M. and Brun, Y.V. (2016) MicrobeJ, a tool for high throughput bacterial cell detection and quantitative analysis. Nat Microbiol, 1, 16077.

30. Wilbanks, E.G., Larsen, D.J., Neches, R.Y., Yao, A.I., Wu, C.-Y., Kjolby, R.A.S. and Facciotti, M.T. (2012) A workflow for genome-wide mapping of archaeal transcription factors with ChIP-seq. Nucleic Acids Research, 40, e74-e74.

31. Langmead, B. and Salzberg, S.L. (2012) Fast gapped-read alignment with Bowtie 2. Nat Methods, 9, 357-359.

32. Li, H., Handsaker, B., Wysoker, A., Fennell, T., Ruan, J., Homer, N., Marth, G., Abecasis, G., Durbin, R. and Genome Project Data Processing, S. (2009) The Sequence Alignment/Map format and SAMtools. Bioinformatics, 25, 2078-2079.

33. Zhang, Y., Liu, T., Meyer, C.A., Eeckhoute, J., Johnson, D.S., Bernstein, B.E., Nusbaum, C., Myers, R.M., Brown, M., Li, W. et al. (2008) Model-based analysis of ChIP-Seq (MACS). Genome Biol, 9, R137.

34. Quinlan, A.R. and Hall, I.M. (2010) BEDTools: a flexible suite of utilities for comparing genomic features. Bioinformatics, 26, 841-842.

35. Lawrence, M., Huber, W., Pages, H., Aboyoun, P., Carlson, M., Gentleman, R., Morgan, M.T. and Carey, V.J. (2013) Software for computing and annotating genomic ranges. PLoS Comput Biol, 9, e1003118. 
36. Koide, T., Reiss, D.J., Bare, J.C., Pang, W.L., Facciotti, M.T., Schmid, A.K., Pan, M., Marzolf, B., Van, P.T., Lo, F.Y. et al. (2009) Prevalence of transcription promoters within archaeal operons and coding sequences. Mol Syst Biol, 5, 285.

37. Taboada, B., Estrada, K., Ciria, R. and Merino, E. (2018) Operon-mapper: a web server for precise operon identification in bacterial and archaeal genomes. Bioinformatics, 34, 4118-4120.

38. Schmid, A.K., Reiss, D.J., Pan, M., Koide, T. and Baliga, N.S. (2009) A single transcription factor regulates evolutionarily diverse but functionally linked metabolic pathways in response to nutrient availability. Mol Syst Biol, 5, 282-282.

39. Tonner, P.D., Pittman, A.M.C., Gulli, J.G., Sharma, K. and Schmid, A.K. (2015) A Regulatory Hierarchy Controls the Dynamic Transcriptional Response to Extreme Oxidative Stress in Archaea. PLOS Genetics, 11, e1004912.

40. Quinlan, A.R. and Hall, I.M. (2010) BEDTools: a flexible suite of utilities for comparing genomic features. Bioinformatics, 26, 841-842.

41. Anders, S., Pyl, P.T. and Huber, W. (2015) HTSeq--a Python framework to work with high-throughput sequencing data. Bioinformatics, 31, 166-169.

42. Chen, X., Zhang, B., Wang, T., Bonni, A. and Zhao, G. (2020) Robust principal component analysis for accurate outlier sample detection in RNA-Seq data. BMC Bioinformatics, 21, 269.

43. Love, M.I., Huber, W. and Anders, S. (2014) Moderated estimation of fold change and dispersion for RNA-seq data with DESeq2. Genome Biology, 15, 550.

44. Benjamini, Y. and Hochberg, Y. (1995) Controlling the False Discovery Rate: A Practical and Powerful Approach to Multiple Testing. Journal of the Royal Statistical Society. Series $B$ (Methodological), 57, 289-300.

45. Kassambara, A.M., Fabian. (2020). R package version 1.0.7 ed.

46. Wickham, H. (2016) ggplot2: elegant graphics for data analysis. Springer Verlag, New York, NY.

47. Kolde, R. (2019). R package version 1.0.12 ed.

48. Makarova, K.S., Wolf, Y.I. and Koonin, E.V. (2015) Archaeal Clusters of Orthologous Genes (arCOGs): An Update and Application for Analysis of Shared Features between Thermococcales, Methanococcales, and Methanobacteriales. Life, 5.

49. Eun, Y.J., Ho, P.Y., Kim, M., LaRussa, S., Robert, L., Renner, L.D., Schmid, A., Garner, E. and Amir, A. (2018) Archaeal cells share common size control with bacteria despite noisier growth and division. Nat Microbiol, 3, 148-154.

50. Vauclare, P., Natali, F., Kleman, J.P., Zaccai, G. and Franzetti, B. (2020) Surviving salt fluctuations: stress and recovery in Halobacterium salinarum, an extreme halophilic Archaeon. Sci Rep, 10, 3298.

51. Zerulla, K. and Soppa, J. (2014) Polyploidy in haloarchaea: advantages for growth and survival. Front Microbiol, 5, 274.

52. Stevenson, K., McVey, A.F., Clark, I.B.N., Swain, P.S. and Pilizota, T. (2016) General calibration of microbial growth in microplate readers. Scientific Reports, 6, 38828.

53. Coker, J.A., DasSarma, P., Kumar, J., Muller, J.A. and DasSarma, S. (2007) Transcriptional profiling of the model Archaeon Halobacterium sp. NRC-1: responses to changes in salinity and temperature. Saline Syst, 3, 6.

54. Martinez-Pastor, M., Lancaster, W.A., Tonner, P.D., Adams, M.W.W. and Schmid, A.K. (2017) A transcription network of interlocking positive feedback loops maintains intracellular iron balance in archaea. Nucleic Acids Res, 45, 9990-10001.

55. Kaur, A., Pan, M., Meislin, M., Facciotti, M.T., El-Gewely, R. and Baliga, N.S. (2006) A systems view of haloarchaeal strategies to withstand stress from transition metals.

Genome Res, 16, 841-854. 
56. Outten, F.W. (2015) Recent advances in the Suf Fe-S cluster biogenesis pathway: Beyond the Proteobacteria. Biochim Biophys Acta, 1853, 1464-1469.

57. Burrell, M., Hanfrey, C.C., Kinch, L.N., Elliott, K.A. and Michael, A.J. (2012) Evolution of a novel lysine decarboxylase in siderophore biosynthesis. Mol Microbiol, 86, 485-499.

58. Andrei, A.S., Banciu, H.L. and Oren, A. (2012) Living with salt: metabolic and phylogenetic diversity of archaea inhabiting saline ecosystems. FEMS Microbiol Lett, 330, 1-9.

59. Gonzalez, O., Gronau, S., Pfeiffer, F., Mendoza, E., Zimmer, R. and Oesterhelt, D. (2009) Systems analysis of bioenergetics and growth of the extreme halophile Halobacterium salinarum. PLoS Comput Biol, 5, e1000332.

60. Schmid, A.K., Reiss, D.J., Kaur, A., Pan, M., King, N., Van, P.T., Hohmann, L., Martin, D.B. and Baliga, N.S. (2007) The anatomy of microbial cell state transitions in response to oxygen. Genome Res, 17, 1399-1413.

61. Schmid, A.K., Pan, M., Sharma, K. and Baliga, N.S. (2011) Two transcription factors are necessary for iron homeostasis in a salt-dwelling archaeon. Nucleic Acids Res, 39, 2519-2533.

62. Falb, M., Muller, K., Konigsmaier, L., Oberwinkler, T., Horn, P., von Gronau, S., Gonzalez, O., Pfeiffer, F., Bornberg-Bauer, E. and Oesterhelt, D. (2008) Metabolism of halophilic archaea. Extremophiles, 12, 177-196.

63. Todor, H., Gooding, J., Ilkayeva, O.R. and Schmid, A.K. (2015) Dynamic Metabolite Profiling in an Archaeon Connects Transcriptional Regulation to Metabolic Consequences. PLoS One, 10, e0135693.

64. Mescher, M.F. and Strominger, J.L. (1976) Structural (shape-maintaining) role of the cell surface glycoprotein of Halobacterium salinarium. Proc Natl Acad Sci U S A, 73, 26872691.

65. Todor, H., Dulmage, K., Gillum, N., Bain, J.R., Muehlbauer, M.J. and Schmid, A.K. (2014) A transcription factor links growth rate and metabolism in the hypersaline adapted archaeon Halobacterium salinarum. Mol Microbiol, 93, 1172-1182.

66. de Silva, R.T., Abdul-Halim, M.F., Pittrich, D.A., Brown, H.J., Pohlschroder, M. and Duggin, I.G. (2021) Improved growth and morphological plasticity of Haloferax volcanii. Microbiology (Reading), 167.

67. Bonneau, R., Facciotti, M.T., Reiss, D.J., Schmid, A.K., Pan, M., Kaur, A., Thorsson, V., Shannon, P., Johnson, M.H., Bare, J.C. et al. (2007) A predictive model for transcriptional control of physiology in a free living cell. Cell, 131, 1354-1365.

68. Facciotti, M.T., Reiss, D.J., Pan, M., Kaur, A., Vuthoori, M., Bonneau, R., Shannon, P., Srivastava, A., Donohoe, S.M., Hood, L.E. et al. (2007) General transcription factor specified global gene regulation in archaea. Proc Natl Acad Sci U S A, 104, 4630-4635.

69. Baliga, N.S., Goo, Y.A., Ng, W.V., Hood, L., Daniels, C.J. and DasSarma, S. (2000) Is gene expression in Halobacterium NRC-1 regulated by multiple TBP and TFB transcription factors? Mol Microbiol, 36, 1184-1185.

70. Martinez-Pastor, M., Tonner, P.D., Darnell, C.L. and Schmid, A.K. (2017) Transcriptional Regulation in Archaea: From Individual Genes to Global Regulatory Networks. Annu Rev Genet, 51, 143-170.

71. Shimada, T., Ishihama, A., Busby, S.J.W. and Grainger, D.C. (2008) The Escherichia coli RutR transcription factor binds at targets within genes as well as intergenic regions. Nucleic Acids Research, 36, 3950-3955.

72. Dorman, C.J., Schumacher, M.A., Bush, M.J., Brennan, R.G. and Buttner, M.J. (2020) When is a transcription factor a NAP? Curr Opin Microbiol, 55, 26-33. 
bioRxiv preprint doi: https://doi.org/10.1101/2021.10.14.464415; this version posted October 14, 2021. The copyright holder for this preprint (which was not certified by peer review) is the author/funder, who has granted bioRxiv a license to display the preprint in perpetuity. It is made available under aCC-BY-NC-ND 4.0 International license. 


\section{FIGURE LEGENDS}

Figure 1: The $\triangle$ hpyA strain is impaired for growth under reduced salt conditions. (A) Spline-smoothed growth curves for the $\Delta$ ura3 parent ("WT", blue curves) compared to the $\triangle$ hpyA mutant (red curves) under optimal salt (dark colors) and reduced salt (light colors). For each strain under each condition, the mean of 9 biological replicate growth curves is shown with surrounding shaded region representing the $99 \%$ confidence interval $(\mathrm{Cl}$, in some curves, shading is not visible because the mean line and $\mathrm{Cl}$ overlap). (B) Dot plots of relative maximum instantaneous growth rate $\left(\mu_{\text {max }}\right)$ for each of the WT and $\Delta h p y A$ strains. Each dot represents one of nine biological replicate trials measuring the $\mu_{\max }$ for each strain under reduced salt compared with its own growth in optimal conditions. Horizontal bars represent the median of each distribution.

Figure 2: Circularity of $\mathbf{H b t}$. salinarum increases when hpyA is deleted under reduced salt. In dot plot, dots represent circularity measurements of individual cells. Horizontal bars are the median of the distribution in each strain under each condition. Shaded regions represent the 95\% bias-corrected confidence interval from bootstrap resampling (see Methods). Below, representative micrograph images are shown for cells of WT, $\triangle h p y A$, and complemented strain $(\Delta h p y A+h p y A$, i.e. pKAD103, Table S2) cells in optimal and reduced salt media. Scale bar is 5 $\mu \mathrm{m}$ and consistent across images. Colors are as in Figure 1. Number of cells counted: WT in optimal salt, $\mathrm{n}=363$; WT in reduced salt, $\mathrm{n}=383$; $\triangle h p y A$ in optimal salt, $\mathrm{n}=188 ; \Delta h p y A$ in reduced salt, 187; complemented strain in optimal salt, $n=313$; complemented strain in reduced salt, $n=360$.

Figure 3: ChIP-seq of HpyA shows salt and growth phase dependent binding patterns. (A) Chromosome-wide binding pattern (measured as read-depth of IP/Input) of HpyA-HA in optimal salt and exponential growth phase (red), optimal salt and stationary phase (pink), reduced salt and exponential phase (dark blue), reduced salt and stationary phase (light blue). (B) Reproducible peaks detected across at least 2 of 4 biological replicates for each condition shorter peaks represent those found in 2 replicates only, while peak at full heights were detected in at least 3 replicates for that particular condition. Note that peaks shown in tag-alone control have been removed from the other conditions and from further analysis. (C) Venn diagram indicating the number of peaks detected in the different conditions. Circles are not scaled by number of peaks.

Figure 4: HpyA binds without preference for coding vs non-coding regions. Height of the bar graph corresponds to percentage of ChIP-seq peaks in non-coding (intergenic, promoter) regions of the genome. Color of the bars are shaded by negative log10 $p$-value of enrichment of peak locations in promoter regions (see scale at right). Actual $p$-values of enrichment calculated by hypergeometric test for each TF are also written below each bar. HpyA binding locations (left) are compared with those for characterized TFs TrmB and RosR in Hbt. salinarum (Hbt) $(38,39)$.

Figure 5: HpyA regulates gene expression in a salt-dependent manner. (A) Venn diagram illustrates the number of genes differentially expressed due to knockout of $h p y A$ in different conditions. Genes with significant $\triangle h p y A$ :WT ratios in optimal salt are shown in red, genes with significant $\triangle h p y A$ : WT ratios low salt in pink, genes with significant low : optimal salt ratios in WT in blue. (B) arCOG enrichment of differentially expressed genes. X-axis shows the number of differentially expressed genes in each category that are annotated in the arCOG ontology, $y-$ axis lists the arCOG category functions and short-hand single letter designations. Categories 
enriched in low salt are listed in pink text, categories enriched across conditions in black. Bars are shaded by Benjamini-Hochberg corrected (44) $p$-values of significance of enrichment according to the scale shown in the legend.

Figure 6: HpyA-dependent regulon shows diverse expression patterns in the conditions tested. (A) Clustering heatmap of genes differentially expressed in response to hpyA deletion across both optimal and low salt conditions. Each column corresponds to the genotype in each condition and rows represent the averaged normalized counts for each gene. Each row is selfstandardized for normalization. Genes labeled with certain colors represent gene functional categories (see legend for colors). Dots next to genes represent monocistronic genes, vertical bars indicate differentially expressed operons. (B) Clustering heatmap of genes differentially expressed in response to hpyA deletion in low salt conditions alone. (C) Normalized reads for 3 selected direct targets of HpyA. Each box corresponds to a particular gene target indicated in the heatmap. In each panel, ChIP-seq data are shown in the top box, RNA-seq data in the middle, genomic context at bottom. ChIP-seq y-axes represent the ratio of IP to input control (whole cell extract, or WCE). RNA-seq y-axes represent read depth for WT in reduced salt (grey traces) and $\mathrm{KO}$ in reduced salt (black). Genonmic context images include the differentially expressed gene(s) (black arrows) and neighboring genes (grey arrows). 


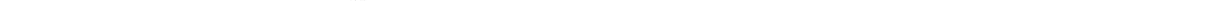


A
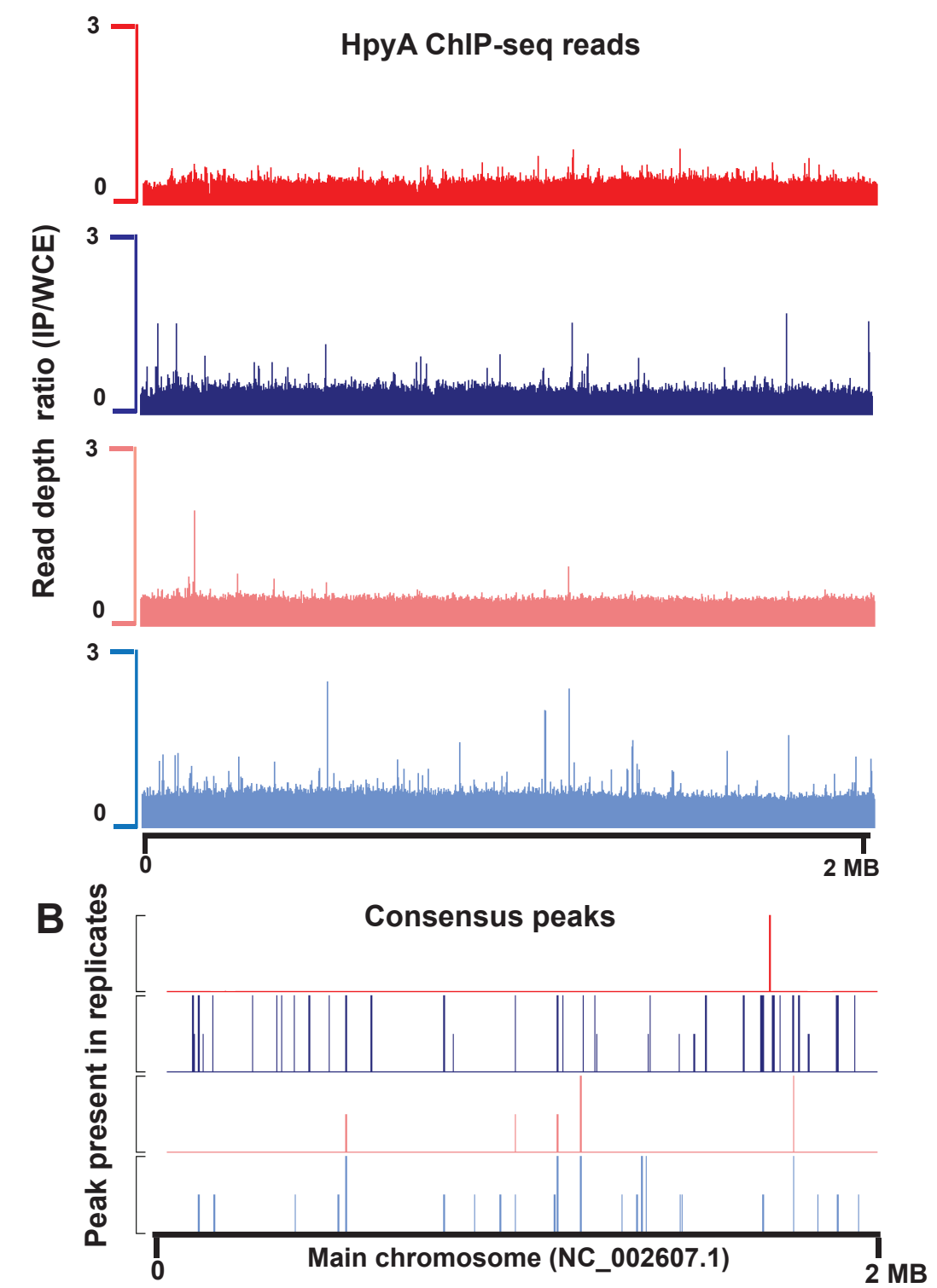

C

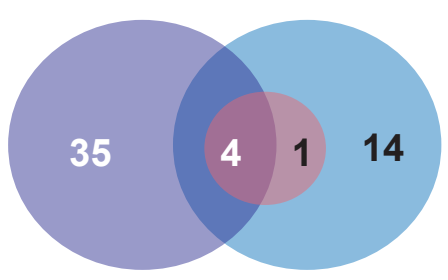

Optimal salt, exponential phase

Low salt, exponential phase

Optimal salt/stationary phase

Low salt/stationary phase 


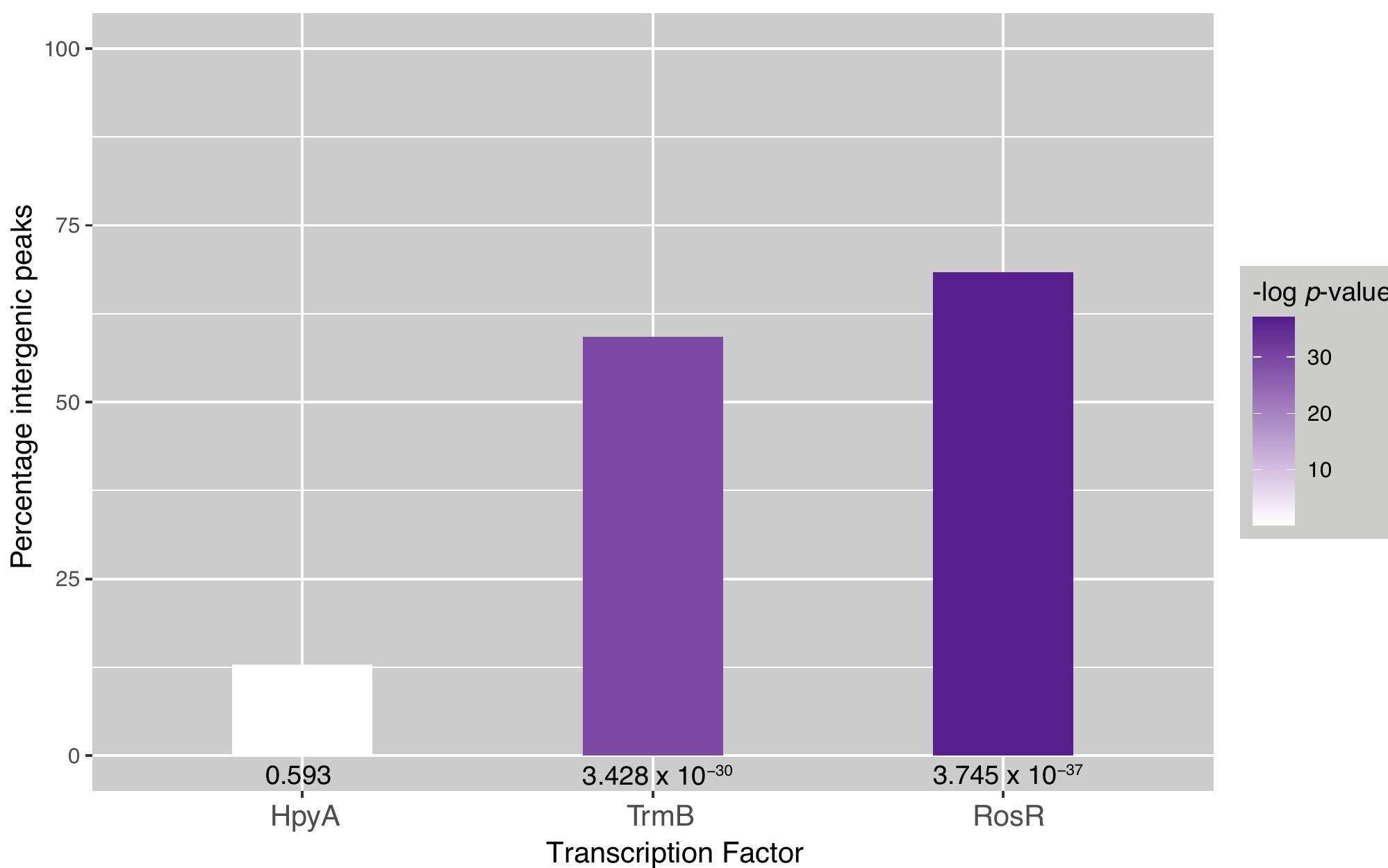




\section{A}

\section{$\triangle$ hpyA:WT low salt $\triangle$ hpyA:WT optimal salt}

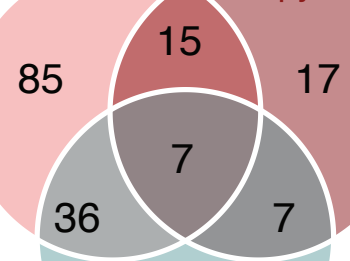

831
B

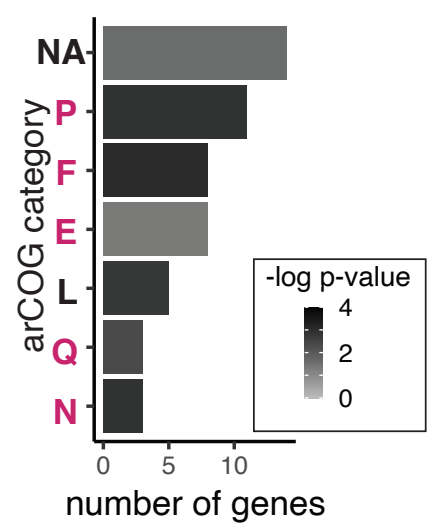

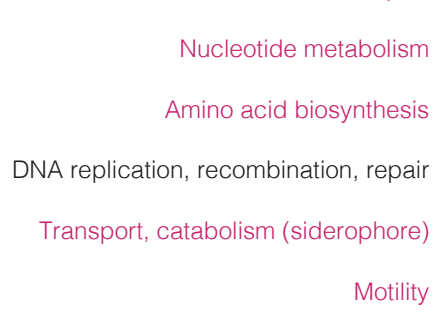

number of genes 
A Optimal + Low salt

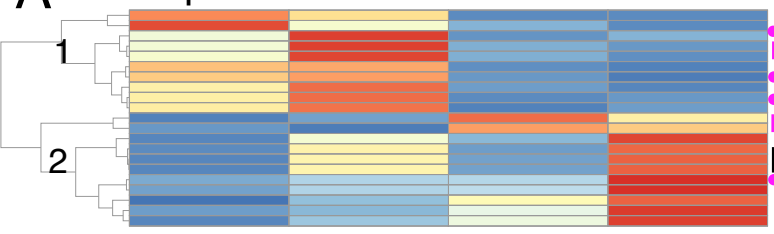

\section{B Low salt cluster 1}

1.1

1.2
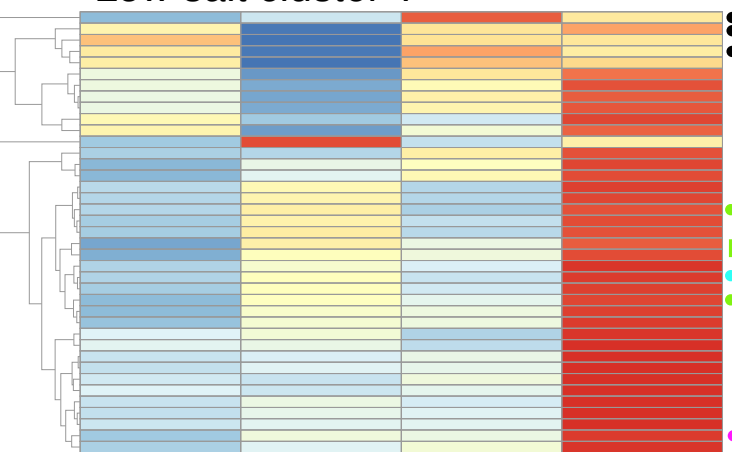

ion transport (iron, metal, chloride)

2.1

2.2

- Ribonucleotide reductase

DNA topoisomerase

- polB2 (DNA polymerase)

radA

Replication protein $\mathrm{A}$

Oligopeptide transport

ssb

helA helicase

\section{Low salt cluster 2}

$\cdot \operatorname{argH}$

iron-binding siderophore

iron

hsmR (multidrug efflux)

ribose / amino acids

Isuf (Fe-S cluster)

VNG0147C (ZntA regulator) - VNG0405C (oxidoreductase)

zurA Mn(II) transport

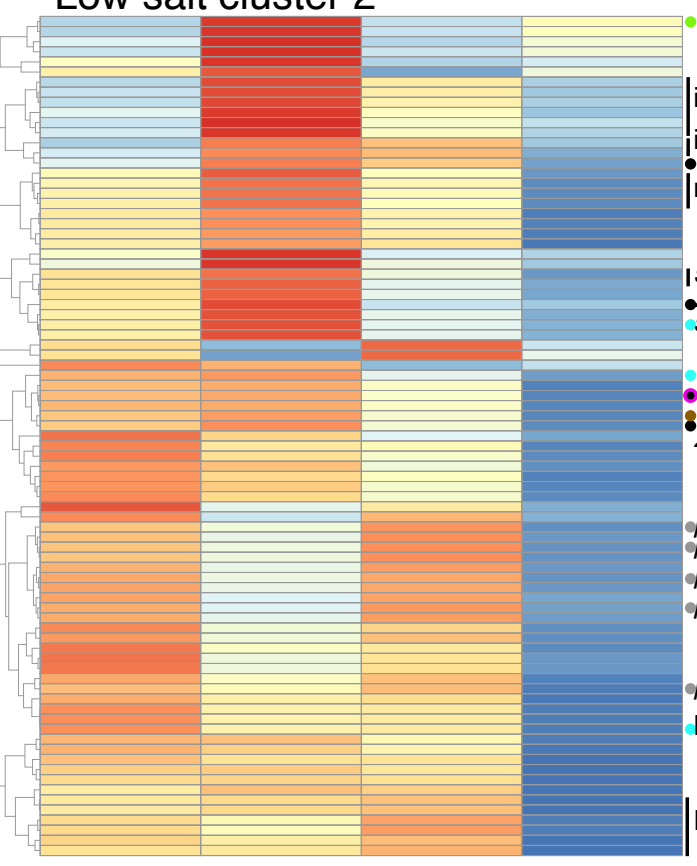
sirt
sirR (heavy metal efflux P-type ATPase)

\section{purE \\ purB \\ purQ \\ purD}

Lrp family transcription factor (trh3)

Phosphate
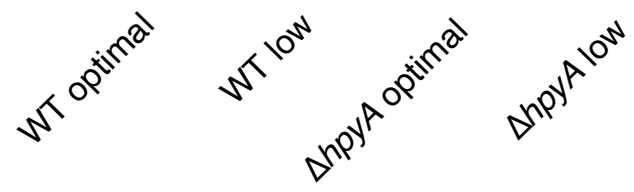

Standardized expression value

$$
1 \quad b_{\text {or }}^{1} \circ \text { ir }
$$

C
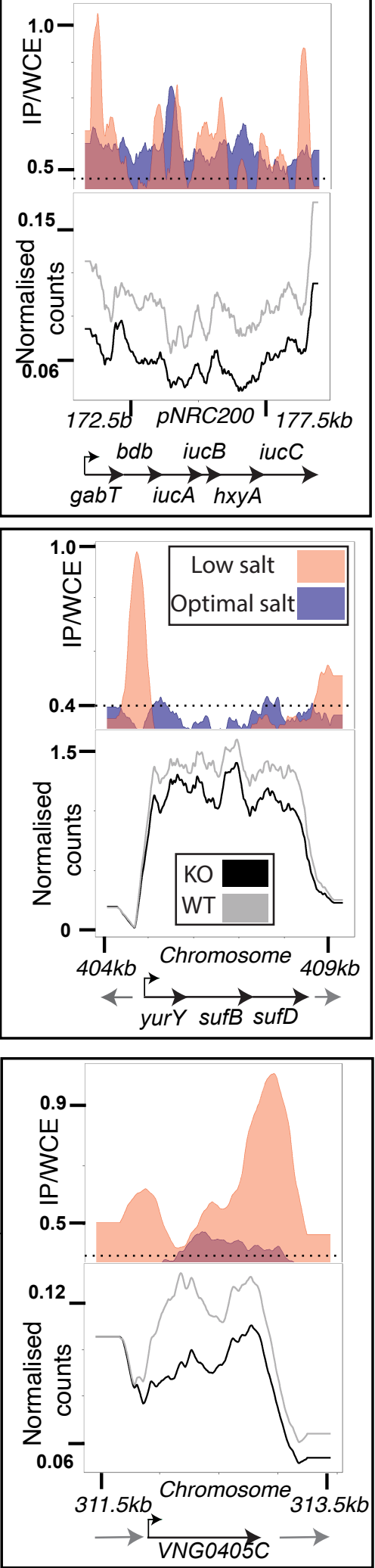